\title{
Low shear stress-induced autophagy alleviates cell apoptosis in HUVECs
}

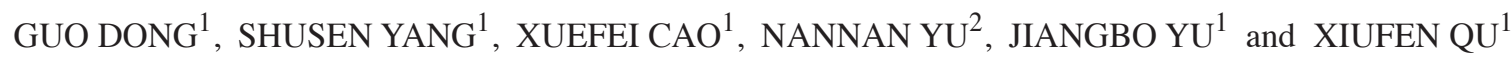 \\ ${ }^{1}$ Cardiovascular Department; ${ }^{2}$ Department of Ophthalmology, The First Affiliated Hospital \\ of Harbin Medical University, Harbin, Heilongjiang 150001, P.R. China
}

Received February 27, 2016; Accepted February 9, 2017

DOI: $10.3892 / \mathrm{mmr} .2017 .6401$

\begin{abstract}
Low shear stress (LSS) is a well-established risk factor resulting in endothelial apoptosis and atherosclerosis. Autophagy has been reported to be involved in the development of atherosclerosis. However, whether autophagy participates in LSS-induced atherosclerosis remains unclear. The effect of autophagy and its association with apoptosis, in the development of atherosclerosis, remains controversial. Therefore, in the present study, the level and role of autophagy in human umbilical vein endothelial cells (HUVECs) exposed to LSS was examined. The results revealed that LSS increased the formation of autophagosomes and MAP1 light chain 3-like protein (LC3) puncta (as demonstrated by transmission electron microscopy and immunofluorescence), and the protein levels of Beclin-1 and LC3II decreased the expression of p62 [as revealed by western blot analysis (WB)]. Furthermore, the level of p62 decreased when autophagy was induced by rapamycin, and increased when autophagy was inhibited by chloroquine (CQ), which indicated that LSS may serve an important role in inducing autophagy flux. In addition, it was observed that HUVECs treated with LSS underwent apoptotic death, by monitoring the rate of apoptosis and the expression of apoptosis regulator BAX (Bax) and apoptosis regulator Bcl-2 (Bcl-2) (by flow cytometry and $\mathrm{WB}$ ) and the LSS-induced apoptosis in HUVECs, that was significantly alleviated by pretreatment with rapamycin, partially via a decrease in the level of Bax and an increase in the level of Bcl-2. Pretreatment of HUVECs with CQ markedly increased LSS-induced apoptosis, which was associated with an increased expression of Bax and a decreased expression of $\mathrm{Bcl}-2$. In conclusion, the results of the present study indicate that LSS increases the level of autophagy, which may be
\end{abstract}

Correspondence to: Mrs. Xiufen Qu, Cardiovascular Department, The First Affiliated Hospital of Harbin Medical University, 23 Youzheng Street, Harbin, Heilongjiang 150001, P.R. China E-mail: xiufenqu@163.com

Key words: low shear stress, atherosclerosis, autophagy, apoptosis, mechanism through a Bcl-2/Beclin-1-dependent mechanism, which serves a protective role against LSS-induced apoptosis.

\section{Introduction}

It is well-known that atherosclerosis preferentially occurs at sites where the blood flow is slow or disturbed, and where the wall shear stress is low or oscillatory (1). Studies have demonstrated that low shear stress (LSS) or oscillatory flow [0-4 Dyne $\left(\right.$ dyn) $\left./ \mathrm{cm}^{2}\right)$, promotes an atherogenic endothelial phenotype with increased endothelial cell proliferation and apoptosis (2), which destroys the endothelium barrier, and initiates an inflammatory response, causing oxidized low-density lipoprotein accumulation in the artery wall and therefore, progression of atherosclerosis (3). The causative association of LSS with atherosclerosis has been demonstrated (4). LSS is a well-established risk factor resulting in atherosclerosis, and LSS is critically important in regulating the vascular physiology and pathology of the vessel walls, by modulating the endothelial cell function (5). However, the detailed molecular mechanisms underlying LSS-induced atherosclerosis remain unclear.

Autophagy is a highly regulated process, that may be involved in the turnover of long-lived proteins and organelles, and may help cells survive in an unfavorable environment (6). Parts of the cytoplasm and intracellular organelles are sequestered within characteristic double-membrane autophagic vacuoles (known as autophagosomes) and are ultimately delivered to lysosomes for bulk degradation. Previously, increasing evidence revealed that autophagy is involved in the pathogenesis of atherosclerosis, stimulated by oxidized lipids, inflammation or metabolic stress $(7,8)$. However, it is unclear whether autophagy participates in the molecular mechanism underlying LSS-induced atherosclerosis. Furthermore, the role of autophagy, either protective or detrimental, in human umbilical vein endothelial cell (HUVEC) death induced by LSS is also poorly understood. In the present study, it was examined whether LSS was able to induce activation of autophagy in HUVECs, and the contribution of autophagy to cell apoptosis and survival under LSS was evaluated.

\section{Materials and methods}

Reagents. Antibodies against MAP1 light chain 3-like protein (LC3; cat. no. L7543), rapamycin (cat. no. V900930), 
chloroquine (CQ; cat. no. C6628) and DAPI (cat. no. D9542) were purchased from Sigma-Aldrich (Merck KGaA, Darmstadt, Germany). Antibodies against apoptosis regulator Bcl-2 (Bcl-2; cat. no. sc7382), apoptosis regulator BAX (Bax; cat. no. sc70408), Beclin-1 (cat. no. sc48381) and $\beta$-actin (cat. no. sc47778) were purchased from Santa Cruz Biotechnology, Inc. (Dallas, TX, USA). Antibody against p62 (cat. no. $5114 \mathrm{~s}$ ) was purchased from Cell Signaling Technology, Inc. (Danvers, MA, USA). IR-Dye 680 (cat. no. 926-32220) or 800cw (cat. no. 926-32211) labeled secondary antibodies were purchased from Li-Cor Biosciences (Lincoln, NE, USA). The HUVECs were provided by the Type Culture Collection of the Chinese Academy of Sciences (Shanghai, China). High-glucose Dulbecco's modified Eagle's medium (DMEM) and fetal bovine serum (FBS) were purchased from Invitrogen (Thermo Fisher Scientific, Inc., Waltham, MA, USA). Annexin-V-fluorescein isothiocyanate (FITC)/propidium iodide (PI) Apoptosis Detection kits were purchased from BD Biosciences (Franklin Lakes, NJ, USA).

Cell culture. The HUVECs were cultured in high-glucose DMEM supplemented with $10 \%$ FBS, in a $95 \%$ humidified incubator, with $5 \% \mathrm{CO}_{2}$ at $37^{\circ} \mathrm{C}$. For all of the experiments, HUVECs in passage 3 were used.

Shear stress experiment. The flow experiments were performed as previously described (9). A parallel-plate flow system was used to impose a laminar shear stress of $1.5 \mathrm{dyn} / \mathrm{cm}^{2}$. The system was maintained at $37^{\circ} \mathrm{C}$ and ventilated with $95 \%$ humidified air containing $5 \% \mathrm{CO}_{2}$.

Treatment of cells with rapamycin and CQ. At 70-80\% confluency, the cells were treated with $5 \mathrm{nM}$ rapamycin or $20 \mu \mathrm{M}$ CQ for $24 \mathrm{~h}$, followed by treatment with LSS $\left(1.5 \mathrm{dyn} / \mathrm{cm}^{2}\right)$ for an additional $0.5,1,2$ or $3 \mathrm{~h}$, respectively. The samples under static conditions (no flow) were used as the control.

Flow cytometry analysis of apoptosis. Apoptosis in the HUVECs was measured with the Annexin V-FITC/PIApoptosis Detection kit, according to the manufacturer's protocol. The stained cells were analyzed by flow cytometry (BD FACSAria III; BD Biosciences, Franklin Lakes, NJ, USA). Data analysis was performed using FlowJo version 7.6.1 (Tree Star, San Carlos, CA, USA).

Transmission electron microscopy (TEM). Cells were seeded at a density $2 \times 10^{5}$ cells/well and fixed in $2.5 \%$ PBS glutaraldehyde at $4^{\circ} \mathrm{C}$ for $1 \mathrm{~h}$. Post-fixation was performed in $1 \% \mathrm{OsO}_{4}$, for $1 \mathrm{~h}$. The cells were dehydrated in an ethanol gradient and embedded in Araldite (Huntsman Co., Ltd., Salt Lake City, UT, USA). Sections (40-60 nm) were placed on a grid (200 mesh) and were double-stained with uranylacetate and lead citrate. The sections were observed under a Philips CM-120 TEM.

Immunofluorescence. The cells were fixed with $4 \%$ paraformaldehyde, permeabilized with $0.2 \%$ Triton X-100, blocked with $5 \%$ non-fat milk for $2 \mathrm{~h}$ at room temperature, incubated with LC3antibodies (1:100) overnight at $4^{\circ} \mathrm{C}$ and stained with DAPI for $1 \mathrm{~h}$, followed by incubation with FITC-conjugated

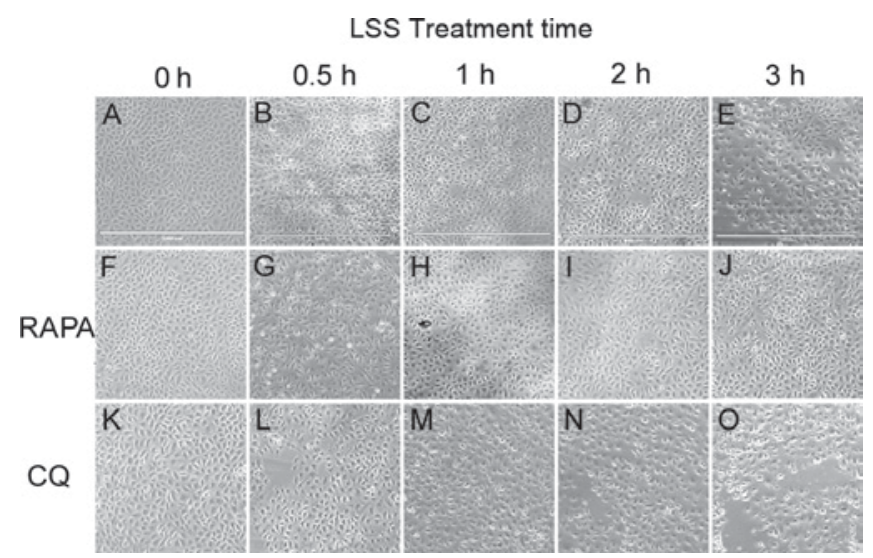

Figure 1. Effects of LSS on the morphology of HUVECs. Cells were maintained under static conditions as controls or subjected to LSS $\left(1.5 \mathrm{dyn} / \mathrm{cm}^{2}\right)$ for (A) 0 , (B) 0.5 , (C) 1, (D) 2 or (E) $3 \mathrm{~h}$. The cells were pretreated with RAPA (5 nM) (f-j) for $24 \mathrm{~h}$ and subjected to LSS $\left(1.5 \mathrm{dyn} / \mathrm{cm}^{2}\right)$ for $(\mathrm{F}) 0$, (G) 0.5 , (H) 1, (I) 2, and (J) $3 \mathrm{~h}$. The cells were pretreated with CQ $(20 \mu \mathrm{M})$ for $24 \mathrm{~h}$ and subjected to LSS $\left(1.5 \mathrm{dyn} / \mathrm{cm}^{2}\right)$ for (K) 0 , (L) $0.5,(\mathrm{M}) 1$, (N) 2 , and $(\mathrm{O}) 3 \mathrm{~h}$. Images of the cellular morphology were captured using a microscope. Scale bar, 1,000 $\mu \mathrm{m}$. HUVEC, human umbilical vein endothelial cells; LSS, low shear stress; RAPA, rapamycin; CQ, chloroquine.

secondary antibody (1:80, cat. no. ZF-0311, Beijing Zhongshan Golden Bridge Biotechnology Co. Ltd., Beijing, China), immunoglobulin $\mathrm{G}$, for $2 \mathrm{~h}$. The images of the cells were captured using a fluorescence microscope (Leica TCS SP5). To quantify autophagic cells, LC3 puncta were determined in triplicate by counting $>30$ cells.

Western blotting (WB). WB was performed as previously described (10). Briefly, cells were washed twice with ice-cold PBS and lysed in ice-cold Western and IP cell protein lysis buffer (Beyotime Institute of Biotechnology, Shanghai, China) containing $20 \mathrm{mM}$ Tris ( $\mathrm{pH} 7.5$ ), $150 \mathrm{mM} \mathrm{NaCl}, 1 \%$ Triton X-100, supplemented with $1 \%(\mathrm{v} / \mathrm{v})$ protease inhibitor cocktail and phenylmethanesulfonyl fluoride (Beyotime Institute of Biotechnology). The extracts were incubated on ice for $30 \mathrm{~min}$, centrifuged at $12,000 \mathrm{x} \mathrm{g}$ for $10 \mathrm{~min}$ at $4^{\circ} \mathrm{C}$ and the supernatants were collected. Protein concentrations were determined with a BCA Protein Assay kit (Beyotime Institute of Biotechnology), and total protein $(20 \mu \mathrm{g})$ was separated by $10-12 \%$ SDS-PAGE, and transferred to nitrocellulose membranes. The membranes were blocked with $5 \%$ non-fat milk for $2 \mathrm{~h}$ at room temperature and incubated overnight at $4^{\circ} \mathrm{C}$ with antibodies specific for Beclin-1 (1:600), LC3 (1:1,000), p62 (1:800), Bax (1:500), Bcl-2 (1:500), and $\beta$-actin $(1: 1,000)$, followed by incubation with goat anti-mouse or goat anti-rabbit IR-Dye 680 or $800 \mathrm{cw}$ labeled secondary antibodies $(1: 10,000)$ for $1 \mathrm{~h}$ at room temperature. Membranes were scanned using a Li-Cor Odyssey scanner. Each band of interest returned near-infrared fluorescent values of raw intensity with intra-lane background subtracted using Odyssey 3.0 analytical software (Li-Cor Biosciences).

Statistical analysis. All of the data were representative of at least three independent experiments and were expressed as the mean \pm standard deviation. Statistical analyses were performed using one-way analysis of variance, followed by the Student-Newman-Keuls test. $\mathrm{P}<0.05$ was considered to 


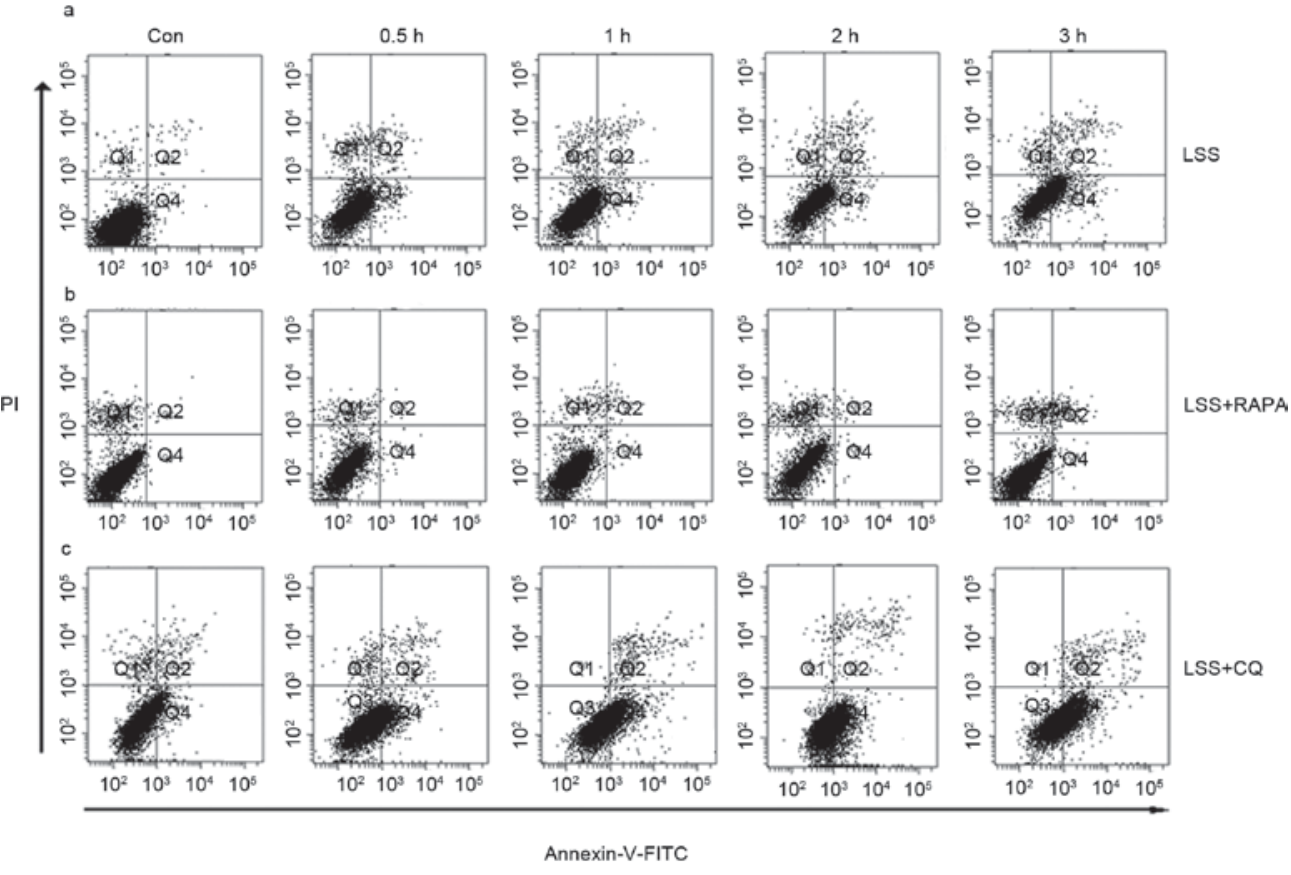

B

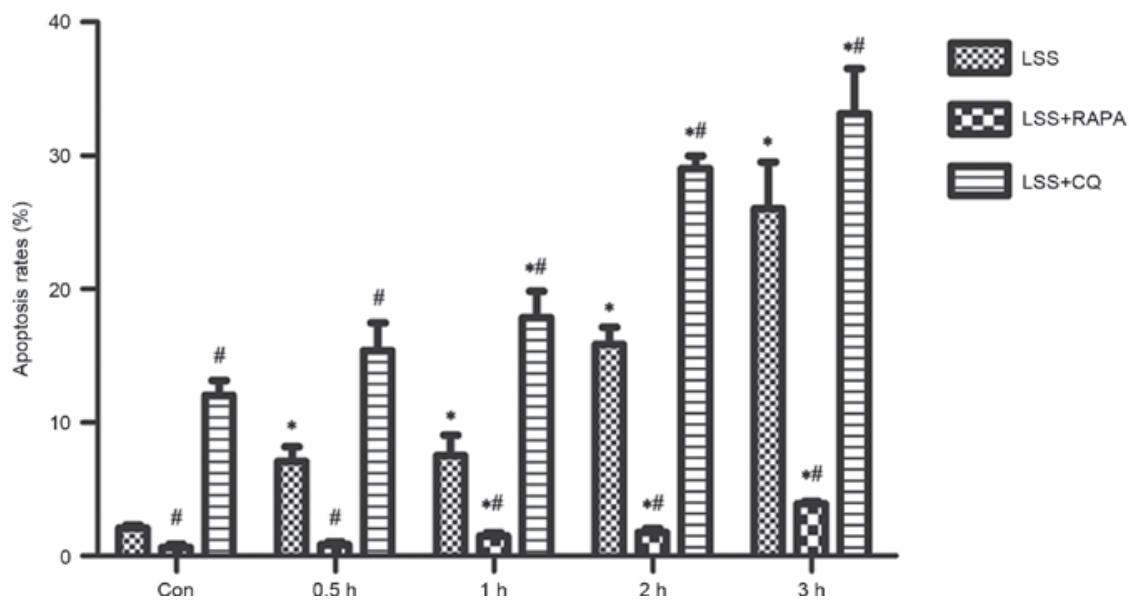

Figure 2. LSS induces the apoptosis of HUVECs. (A) Results of apoptosis assay following treatment with (A-a) LSS, (A-b) RAPA and (A-c) CQ. (B) Quantification of the apoptosis data. The bar graphs represent the means \pm standard error $(\mathrm{n}=3)$. " $\mathrm{P}<0.01$ vs. the control. " $\mathrm{P}<0.01$ vs. the cells pretreated with different modulators at the same point. LSS, low shear stress; HUVEC, human umbilical vein endothelial cells; RAPA, rapamycin; CQ, chloroquine; Con, control; PI, propidium iodide; FITC, fluorescein isothiocyanate.

indicate a statistically significant difference. The statistical analysis was performed using SPSS software (version 18.0; SPSS, Inc., Chicago, Il, USA).

\section{Results}

Cell morphological and viability changes of HUVECs. Increased LSS-only treatment times led to a gradual reduction in cell viability, with cell shrinkage and easy detachment from the coverslip compared with static cells (Fig. 1A-E). Treatment with rapamycin alone exhibited no effect on the morphology of HUVECs in static condition (Fig. 1F), whereas treatment combined with LSS effectively attenuated the cell injury induced by LSS and preserved the shape of the HUVECs compared with LSS group at the same time point (Fig. 1G-J). Treatment with CQ alone had no influence on the morphology of HUVECs in static condition (Fig. 1K), whereas CQ+LSS treatment exacerbated cell shrinkage and the cells detached more easily from the coverslip compared with LSS-only group at the same time points (Fig. 1L-O).

Effects of LSS, LSS $+R A P A$ and $L S S+C Q$ on cell apoptosis of HUVECs. Flow cytometry analysis demonstrated that LSS-only treatment resulted in a significant increase in apoptosis in a time-dependent manner (Fig. 2A-a and B). Rapamycin treatment reduced the pro-apoptotic effect of LSS treatment in HUVECs compared with the control and LSS-only group at the same time points (Fig. 2A-b and B). In addition, CQ led to a significant increase in the later apoptotic cell population and an increase of the early apoptotic cell population compared with the control and LSS-only groups at the same time points (Fig. 2A-c and B). 


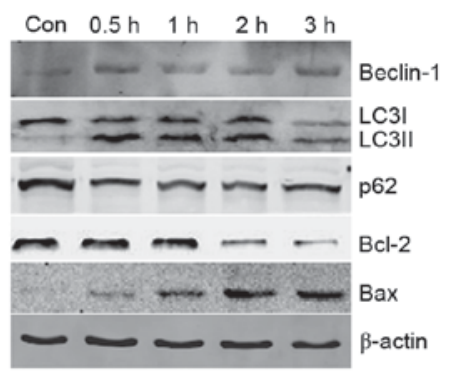

D
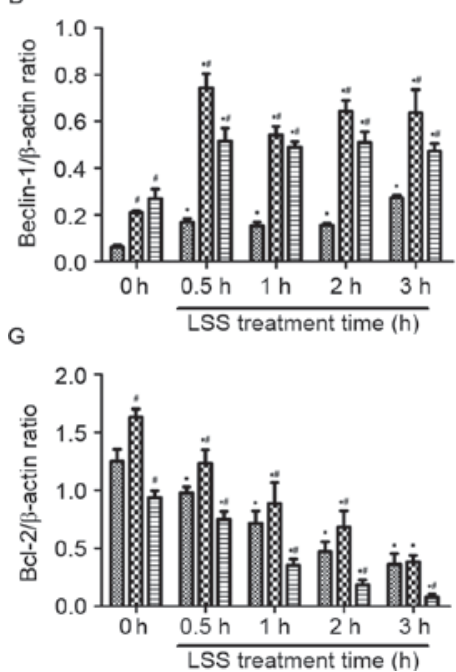

B

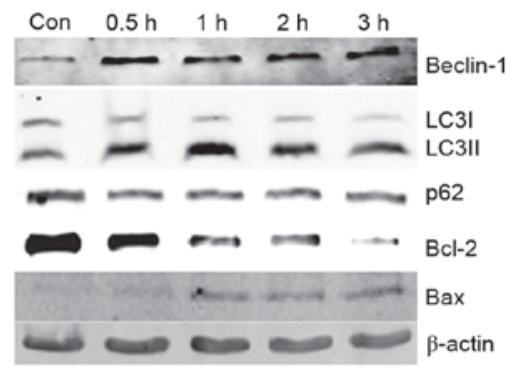

C

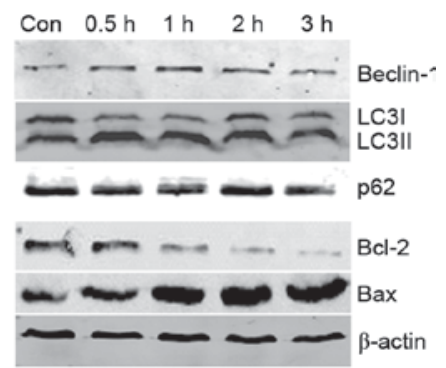

$\mathrm{F}$

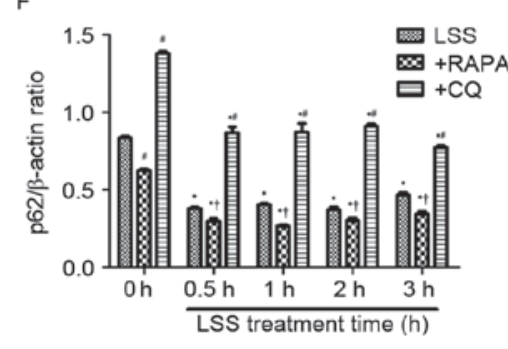

$\mathrm{H}$
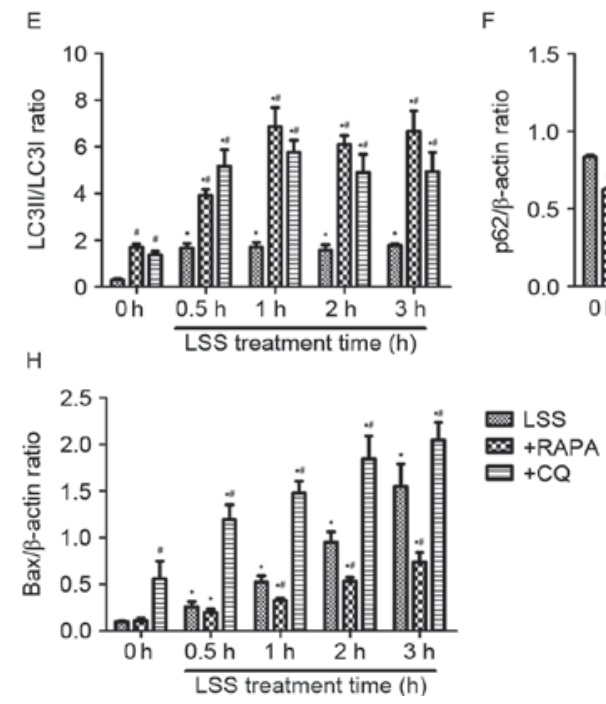

Figure 3. LSS induces autophagy and apoptosis in HUVECs. The expression levels of the Beclin-1, LC3I, LC3II, p62, Bcl-2 and Bax proteins were determined by western blotting, following treatment with (A) LSS, (B) LSS+RAPA and (C) LSS+CQ. (D) Beclin-1 intensity, (E) LC3II/LC3I intensity, (F) p62 intensity, (G) Bcl-2 intensity and (H) Bax intensity were expressed as the fold change between LSS+RAPA, LSS+CQ and LSS. The bar graphs represent the mean \pm standard error $(\mathrm{n}=3)$. ${ }^{*} \mathrm{P}<0.01$ vs. the control. ${ }^{\dagger} \mathrm{P}<0.05$ and ${ }^{\#} \mathrm{P}<0.01$ vs. the cells pretreated with the various modulators at the same point. Con, control; LSS, low shear stress; HUVEC, human umbilical vein endothelial cells; RAPA, rapamycin; CQ, chloroquine; LC3, MAP1 light chain 3-like protein; Bcl-2, apoptosis regulator $\mathrm{Bcl}-2$; Bax, apoptosis regulator $\mathrm{BAX}$.
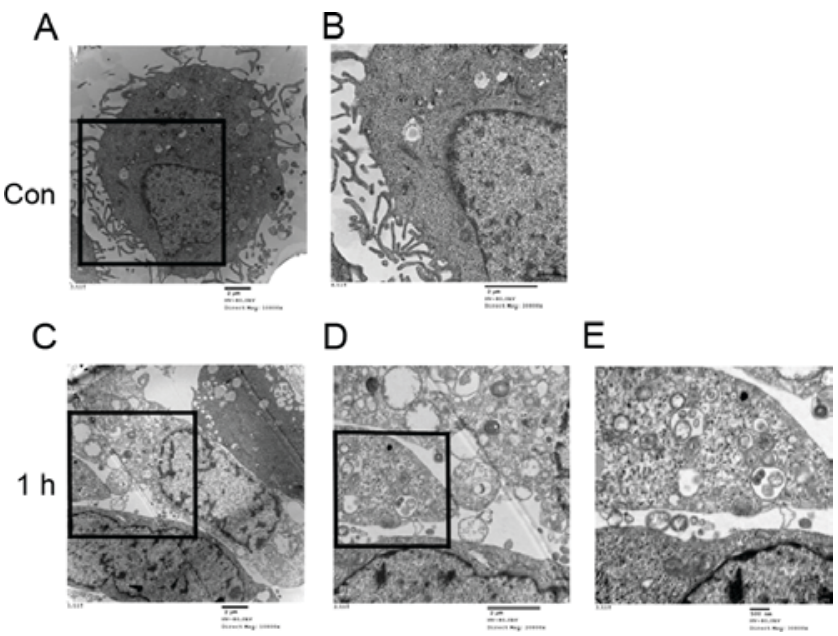

Figure 4. Ultrastructural changes induced by LSS in human umbilical vein endothelial cells. Cells were maintained under static conditions as controls at magnification (A) x10,000 and (B) x20,000, or subjected to LSS (1.5 dyn/cm²) for $1 \mathrm{~h}$ at magnification (C) x10,000, (D) x20,000 and (E) x30,000. The square indicates the study area and is enlarged in the right panel to demonstrate the ultrastructure of the cells. The typical autophagosomes with the characteristic double membrane were shown. LSS, low shear stress; Con, control.

Autophagy and apoptosis-associated protein levels changes in HUVECs treated with LSS, LSS $+R A P A$ or LSS+CQ. Beclin-1, LC3 and p62 proteins are reliable markers for autophagy (Fig. 3). In the present study, the level of Beclin-1 and conversion of LC3I into LC3II were markedly increased, whereas the levels of p62 decreased in the HUVECs treated with LSS for 0.5, 1, 2 and $3 \mathrm{~h}$ compared with control (Fig. 3A and D-F). Rapamycin treatment significantly increased the 
A
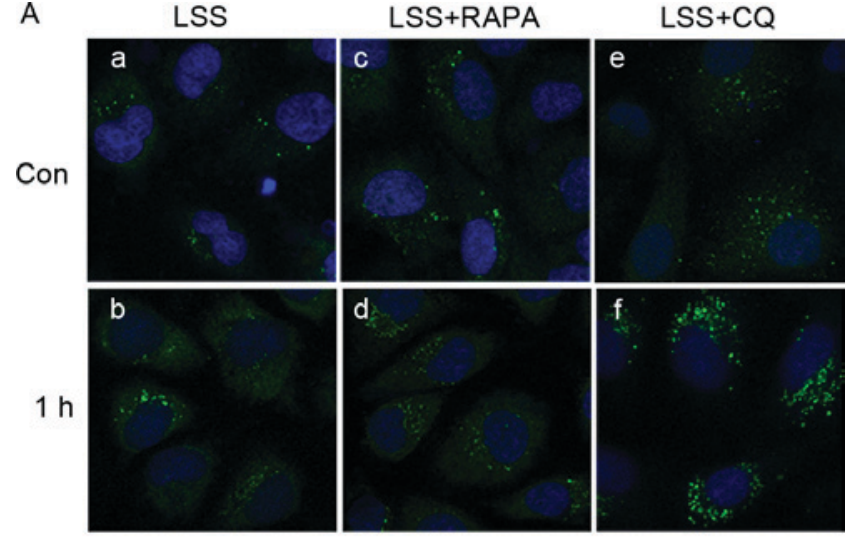

B

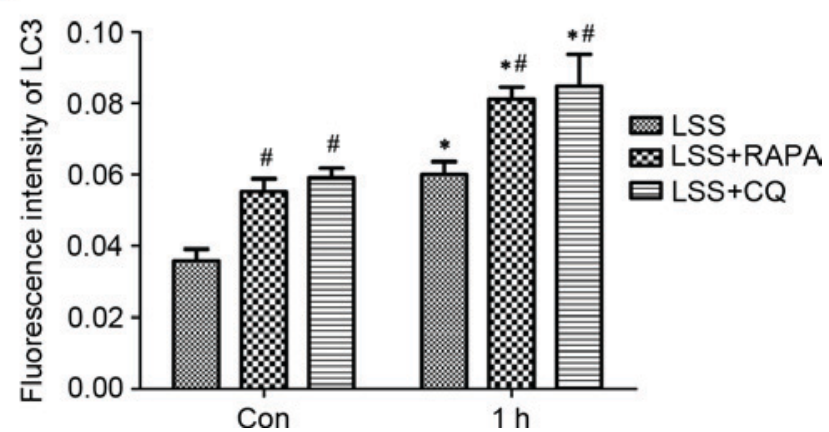

Figure 5. LSS induced autophagosome formation in HUVECs. (A) Cells were maintained under static conditions as controls or subjected to LSS $\left(1.5 \mathrm{dyn} / \mathrm{cm}^{2}\right)$ for (a) $0 \mathrm{~h}$ or (b) $1 \mathrm{~h}$. The cells were pretreated with RAPA $(5 \mathrm{nM})$ for $24 \mathrm{~h}$ and subjected to LSS $\left(1.5 \mathrm{dyn} / \mathrm{cm}^{2}\right.$ ) for (c) $0 \mathrm{~h}$ and (d) $1 \mathrm{~h}$. The cells were pretreated with CQ $(20 \mu \mathrm{M})$ for $24 \mathrm{~h}$ and subjected to LSS $\left(1.5 \mathrm{dyn} / \mathrm{cm}^{2}\right)$ for (e) $0 \mathrm{~h}$ and (f) $1 \mathrm{~h}$. The LC3 puncta (autophagosomes) in the cells were visualized by fluorescence microscopy after immunofluorescence staining with an LC3 antibody, followed by a fluorescein isothiocyanate-labeled secondary antibody (green, original magnification $\mathrm{x} 400$ ). The nucleus was stained with DAPI (blue, original magnification x400). (B) Quantification of fluorescence intensity of LC3. Bar graphs represent average number of typical LC3 puncta/cell. Data are mean \pm standard error from minimum 30 cells for each experiment $(n=3)$. ${ }^{*} \mathrm{P}<0.01$ vs. the control. ${ }^{~} \mathrm{P}<0.01$ vs. the cells pretreated with the various modulators at the same point. LSS, low shear stress; HUVEC, human umbilical vein endothelial cells; Bax, apoptosis regulator BAX; LC3, MAP1 light chain 3-like protein; RAPA, rapamycin; $\mathrm{CQ}$, chloroquine.

level of Beclin-1 and the conversion of LC3I into LC3II (Fig. 3B, D and E); however, the protein expression of p62 was reduced compared with the control and LSS-only groups at the same time points (Fig. 3B and F). LSS+CQ treatment resulted in a significant increase of Beclin-1 expression and the LC3I to LC3II ratio compared with control and LSS group at the same time point (Fig. 3C-E). It also increased p62 expression in HUVECs compared with LSS group at the same time point; however, the p62 expression following LSS+CQ treatment was lower compared with CQ only treatment (Fig. $3 \mathrm{C}$ and F). Bcl-2 and Bax are important members of the Bcl-2 family which have an important role the regulation of apoptosis. In the present study, the expression of Bcl-2 was downregulated and the expression of Bax was upregulated in the LSS treatment group compared with the control group (Fig. 3A, G and H). Compared with RAPA treatment alone, LSS+RAPA treatment for $0.5,1,2$ and $3 \mathrm{~h}$ significantly decreased the Bcl-2 levels (Fig. 3B and G) and increased the Bax levels (Fig. 3B and H). However, compared with LSS groups at the same time points,
LSS+RAPA treatment significantly increased the Bcl-2 levels (Fig. 3B and G) and reduced Bax levels (Fig. 3B and H). CQ treatment increased the Bax levels and reduced the Bcl-2 levels (Fig. 3C, G and H) compared with control and LSS group at the same time point.

Observation of autophagosomes and lysosomes in HUVECs. A normal cytoplasm, mitochondria and nuclei and a small number of autophagosomes and lysosomes were observed in the TEM images of the control group (Fig. 4A and B). HUVECs treated with LSS-only for $1 \mathrm{~h}$ exhibited numerous autophagosomes at various stages of development (Fig. 4C-E).

In addition, the treatment with LSS induced extensive formation of LC3 puncta compared with static cells, as determined by LC3 immunofluorescence staining (Fig. 5A-a, -b and B). Pretreatment with rapamycin significantly increased the formation of the LC3 puncta in HUVECs (Fig. 5A-c, -d and B) compared with control and LSS-only group. Pretreatment with CQ resulted in a significant accumulation of LC3 puncta (Fig. 5A-e, -f and B) in HUVECs compared with control and LSS group.

\section{Discussion}

In the present study, it was demonstrated that atheroprone LSS conditions were able to induce cell autophagy and apoptosis by regulating the balance of $\mathrm{Bcl}-2 / \mathrm{Beclin}-1$ and $\mathrm{Bcl}-2 / \mathrm{Bax}$. The induction of autophagy, by pretreatment with rapamycin, protected the HUVECs against LSS-induced apoptotic cell death. Autophagy inhibition by pretreatment with CQ resulted in elevated apoptotic cell death. With these results, it was concluded that autophagy served an important role in protecting against LSS-induced apoptosis.

Apoptosis is a highly regulated cell death process characterized by cell shrinkage, membrane blebbing, DNA fragmentation and chromatin condensation (11). It constitutes an initial step in endothelial cell dysfunction, which is an important feature in atherosclerosis (12). LSS is a well-established risk factor resulting in atherosclerosis, and it serves a critical role in modulating endothelial cell function. Recently, studies indicate that LSS is able to induce endothelial cell apoptosis (13). Consistent with these previous results, the data from flow cytometry with Annexin-V-FITC/PI dual staining, demonstrated that LSS induced the apoptosis of HUVECs. It is well-known that the Bcl-2 family serves a key role in the process of apoptosis. This family includes anti-apoptotic proteins, including Bcl-2, Bcl-2-like protein 1 and induced myeloid leukemia cell differentiation protein Mcl-1, and pro-apoptotic proteins, including Bax, Bcl-2-associated agonist of cell death and Bcl-2 homologous antagonist/killer (14). A previous study demonstrated that $\mathrm{Bcl}-2 / \mathrm{Bax}$ ratio is a rheostat which determines the incidence of apoptosis (15). In the present study, a marked decrease in Bcl-2 and an increase in Bax in HUVECs treated with LSS was observed. This observation suggests that LSS increases apoptosis by regulating the balance between Bcl-2 and Bax.

Autophagy, another type of programmed cell death, serves an important role in a number of physiological and pathological processes, including aging and cardiac ischemia $(16,17)$. Studies have demonstrated that endothelial cells exhibit 
characteristics of autophagy when the cells are exposed to pro-atherogenic factors (18), which indicates that autophagy may serve a crucial role in regulating the formation and progression of atherosclerosis (7). Beclin-1, LC3 and p62 have been reported as reliable markers of autophagy (19). Beclin-1 was originally identified as a Bcl-2-interacting protein, and was shown to be essential to autophagy. Beclin-1 induces autophagy by interacting with certain cofactors to activate the phosphatidylinositol-3-kinase Vps34 (20). LC3 are essential proteins that regulate the autophagosomal membrane. Under normal conditions, the majority of LC3 proteins present in the cytosol are in the LC3I form. Upon autophagy induction, the cytosolic LC3I form is conjugated with phosphatidylethanolamine and becomes LC3II, which forms a stable association with the autophagosomal membrane (21). p62 (also known as sequestosome 1) serves as an association between LC3 and ubiquitinated substrates to facilitate autophagic clearance. p62 decreases when autophagy is induced, and accumulates when autophagy is inhibited. Therefore, p62 is used as a readout of autophagic degradation and a marker of autophagy flux $(22,23)$. In the present study, an increase in double-membrane autophagosomes by TEM and LC3 puncta was observed by fluorescence microscopy in HUVECs treated with LSS. Monitoring the levels of autophagic proteins revealed an increase in Beclin-1 and LC3II, but an opposite trend in p62 in the LSS-treated HUVECs. Additionally, the level of p62 was downregulated by rapamycin by upregulating the protein level of LC3II. CQ, an inhibitor of autophagy, was able to disrupt the fusion of autophagosomes with lysosomes and suppress the activity of lysosomal acid hydrolases as a weak base, thereby blocking the degradation of autolysosome and accumulating LC3II at a late stage, exhibiting anti-autophagic characteristics (24). Therefore, CQ up-regulated the level of LC3II and p62 by blocking autophagy. This observation indicated that LSS induced autophagy flux, which in turn proved that an autophagy process was activated by pro-atherogenic LSS.

Although Bcl-2 family proteins were initially characterized as cell death regulators, it has recently become clear that they also control autophagy. A study indicated that autophagy induction correlated with the dissociation of Bcelin-1 from Bcl-2 (25). In normal, Beclin-1 is bound to Bcl-2 through interaction involving Bcl-2 homology 3 (BH3) domain in Beclin-1 and the BH3 binding groove of Bcl-2 (20). Phosphorylation of Bcl-2 can lead to Bcl-2 separating from Beclin-1, thereby alleviating the inhibitory effect on Beclin-1 (25). In the present study, we observed that the LSS treatment induced HUVEC autophagy with decreased Bcl-2 levels and increased Beclin-1 levels, indicating that LSS is able to alter the balance between Bcl-2 and Beclin-1 which may be the mechanism of autophagy induced by LSS.

Although the upregulation of autophagy has been observed in HUVECs treated with LSS in the present study, it is unclear whether the autophagy is protective or detrimental. The cross talk between the autophagic and apoptotic cell death pathways is complex (26). The theory that autophagy is initiated as a protective response has become accepted (27). To investigate the effect of autophagy on the LSS-induced apoptosis in HUVECs, rapamycin, a mammalian target of rapamycin (mTOR) inhibitor, was used to induce autophagy. The results demonstrated that rapamycin upregulated the level of autophagy and downregulated the apoptosis rate. Similarly, previous studies demonstrated that rapamycin was able to reduce tert-butyl hydroperoxide-induced apoptosis (28) and mechanical stress-induced endothelial apoptosis (29). Furthermore, rapamycin upregulated the level of Bcl-2, but failed to inhibit the expression of Beclin-1. This may be due to the fact that inhibition of mTOR promotes Beclin-1 expression and prevents the decreasing of Beclin-1 (30). To further investigate the association between autophagy and apoptosis induced by LSS, CQ was used to inhibit autophagy and investigate changes in apoptotic cell death. In the present study, with the decreased level of autophagy induced in CQ, the rate of apoptosis in the HUVECs treated with LSS increased significantly. These results are consistent with the data demonstrating that an increased level of autophagy protects HUVECs from LSS-induced apoptosis, whereas a deceased level of autophagy led to increased apoptosis in HUVECs, which suggests that LSS-induced apoptosis is regulated by autophagy. Autophagy serves a protective role in LSS-induced apoptosis.

The results of the present study suggest that LSS was able to induce autophagy through the modulation of Bcl-2/Beclin-1 in HUVECs. Furthermore, it was observed that the cross talk between autophagy and apoptosis contributes to the autophagic protection of HUVECs from LSS-induced apoptosis. Although these results represent an advancement in the understanding of the association between LSS-induced autophagy and apoptosis, additional work is necessary to further characterize the protective effect of autophagy in the progression of atherosclerosis induced by LSS.

\section{Acknowledgments}

The authors would like to thank Dr Chunlai Wang (Harbin Veterinary Research Institute, Harbin, China) and Professor Zuyan Liu (Harbin Institute of Technology, Harbin, China) for their technical assistance. The present study was supported by the Natural Science Foundation of Heilongjiang Province Project (grant no. H201345) and by the Postdoctoral Foundation of Heilongjiang Province Project (grant no. LBH-Z11062).

\section{References}

1. Farmakis TM, Soulis JV, Giannoglou GD, Zioupos GJ and Louridas GE: Wall shear stress gradient topography in the normal left coronary arterial tree: Possible implications for atherogenesis. Curr Med Res Opin 20: 587-596, 2004.

2. Davies PF, Polacek DC, Shi C and Helmke BP: The convergence of haemodynamics, genomics, and endothelial structure in studies of the focal origin of atherosclerosis. Biorheology 39: 299-306, 2002.

3. Kinlay S and Ganz P: Role of endothelial dysfunction in coronary artery disease and implications for therapy. Am J Cardiol 80: 11I-16I, 1997.

4. Stone PH, Saito S, Takahashi S, Makita Y, Nakamura S, Kawasaki T, Takahashi A, Katsuki T, Nakamura S, Namiki A, et al: Prediction of progression of coronary artery disease and clinical outcomes using vascular profiling of endothelial shear stress and arterial plaque characteristics: The PREDICTION study. Circulation 126: 172-181, 2012.

5. Cunningham KS and Gotlieb AI: The role of shear stress in the pathogenesis of atherosclerosis. Lab Invest 85: 9-23, 2005.

6. Levine B and Kroemer G: Autophagy in the pathogenesis of disease. Cell 132: 27-42, 2008.

7. Martinet W and De Meyer GR: Autophagy in atherosclerosis: A cell survival and death phenomenon with therapeutic potential. Circ Res 104: 304-317, 2009. 
8. Schrijvers DM, De Meyer GR and Martinet W: Autophagy in atherosclerosis: A potential drug target for plaque stabilization. Arterioscler Thromb Vasc Biol 31: 2787-2791, 2011.

9. Levesque MJ and Nerem RM: The elongation and orientation of cultured endothelial cells in response to shear stress. J Biomech Eng 107: 341-347, 1985

10. Yu W, Gu K, Yu Z, Yuan D, He M, Ma N, Lai S, Zhao J, Ren Z, Zhang X, et al: Sorafenib potentiates irradiation effect in hepatocellular carcinoma in vitro and in vivo. Cancer Lett 329: 109-117, 2013.

11. Kerr JF, Wyllie AH and Currie AR: Apoptosis: A basic biological phenomenon with wide-ranging implications in tissue kinetics. Br J Cancer 26: 239-257, 1972

12. Gimbrone MA Jr, Topper JN, Nagel T, Anderson KR and Garcia-Cardeña G: Endothelial dysfunction, hemodynamic forces, and atherogenesis. Ann N Y Acad Sci 902: 230-240, 2000

13. Zhang J, Wang Z, Zhang J, Zuo G, Li B, Mao W and Chen S: Rapamycin attenuates endothelial apoptosis induced by low shear stress via mTOR and sestrin1 related redox regulation. Mediators Inflamm 2014: 769608, 2014.

14. Lin HC and Lai IR: Isolated mitochondria infusion mitigates ischemia-reperfusion injury of the liver in rats: Reply. Shock 39 $543,2013$.

15. Korsmeyer SJ: BCL-2 gene family and the regulation of programmed cell death. Cancer Res 59 (7 Suppl): 1693s-1700s, 1999.

16. Nowicki M, Zabirnyk O, Duerrschmidt N, Borlak J and Spanel-Borowski K: No upregulation of lectin-like oxidized low-density lipoprotein receptor-1 in serum-deprived EA.hy926 endothelial cells under oxLDL exposure, but increase in autophagy. Eur J Cell Biol 86: 605-616, 2007.

17. Khan MJ, Rizwan Alam M, Waldeck-Weiermair M, Karsten F, Groschner L, Riederer M, Hallström S, Rockenfeller P, Konya V, Heinemann A, et al: Inhibition of autophagy rescues palmitic acid-induced necroptosis of endothelial cells. J Biol Chem 287: 21110-21120, 2012.

18. Xie Y, You SJ, Zhang YL, Han Q, Cao YJ, Xu XS, Yang YP, Li J and Liu CF: Protective role of autophagy in AGE-induced early injury of human vascular endothelial cells. Mol Med Rep 4: 459-464, 2011
19. Mizushima N, Yoshimori T and Levine B: Methods in mammalian autophagy research. Cell 140: 313-326, 2010.

20. Levine B, Sinha S and Kroemer G: Bcl-2 family members: Dual regulators of apoptosis and autophagy. Autophagy 4: 600-606, 2008.

21. He C and Klionsky DJ: Regulation mechanisms and signaling pathways of autophagy. Annu Rev Genet 43: 67-93, 2009.

22. Bjørkøy G,Lamark T,Brech A, Outzen H,Perander M,Overvatn A, Stenmark H and Johansen T: p62/SQSTM1 forms protein aggregates degraded by autophagy and has a protective effect on huntingtin-induced cell death. J Cell Biol 171: 603-614, 2005.

23. Pankiv S, Clausen TH, Lamark T, Brech A, Bruun JA, Outzen H, Øvervatn A, Bjørkøy G and Johansen T: p62/SQSTM1 binds directly to Atg8/LC3 to facilitate degradation of ubiquitinated protein aggregates by autophagy. J Biol Chem 282: 24131-24145, 2007.

24. Klionsky DJ, Abeliovich H, Agostinis P, Agrawal DK, Aliev G, Askew DS, Baba M, Baehrecke EH, Bahr BA, Ballabio A, et al: Guidelines for the use and interpretation of assays for monitoring autophagy in higher eukaryotes. Autophagy 4: 151-175, 2008.

25. Pattingre S, Tassa A, Qu X, Garuti R, Liang XH, Mizushima N, Packer M, Schneider MD and Levine B: Bcl-2 antiapoptotic proteins inhibit Beclin 1-dependent autophagy. Cell 122: 927-939, 2005

26. Eisenberg-Lerner A, Bialik S, Simon HU and Kimchi A: Life and death partners: Apoptosis, autophagy and the cross-talk between them. Cell Death Differ 16: 966-975, 2009.

27. Yan WJ, Dong HL and Xiong LZ: The protective roles of autophagy in ischemic preconditioning. Acta Pharmacol Sin 34: 636-643, 2013

28. Shin YJ, Cho DY, Chung TY, Han SB, Hyon JY and Wee WR: Rapamycin reduces reactive oxygen species in cultured human corneal endothelial cells. Curr Eye Res 36: 1116-1122, 2011.

29. Raaz U, Kuhn H, Wirtz H and Hammerschmidt S: Rapamycin reduces high-amplitude, mechanical stretch-induced apoptosis in pulmonary microvascular endothelial cells. Microvasc Res 77: 297-303, 2009.

30. Yang T, Li D, Liu F, Qi L, Yan G and Wang M: Regulation on Beclin-1 expression by mTOR in CoCl2-induced HT22 cell ischemia-reperfusion injury. Brain Res 1614: 60-66, 2015. 